Goldschmidt 2021 Abstract

https://doi.org/10.7185/gold2021.8142

\section{A high resolution multimedia environmental monitoring project at regional and local scale: the Campania region (southern Italy) case study}

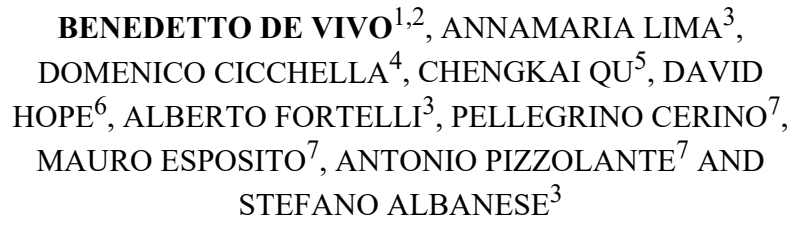

${ }^{1}$ Virginia Tech

${ }^{2}$ Pegaso On-Line University (Italy)

${ }^{3}$ University of Naples Federico II

${ }^{4}$ University of Sannio

${ }^{5}$ China University of Geosciences

${ }^{6}$ Pacific Rim lab

${ }^{7}$ Istituto Zooprofilattico Sperimentale del Mezzogiorno

Presenting Author: devivob@libero.it

Campania region, southern Italy, in the last decade faced environmental issues whose entity was badly perceived by public due to a strong scaremonger mediatic pressure and a substantial lack of organic scientific data on the environmental conditions of the territory.

To generate a clear picture of the situation and to limit the extent of damages to the economy of agri-food sector, in 2015 Campania regional government funded the multipurpose project "Campania Trasparente" assigning a management role to Istituto Zooprofilattico Sperimentale del Mezzogiorno (IZSM). IZSM with the support of several researchers, including the authors, completed a wide survey on regional territory to: a) determine concentrations of inorganic and organic chemicals in soils, air, vegetable crops and groundwaters; b) define the degree of bioavailability of toxic elements and compounds in soil; c) determine the transfer rate of contaminant from soil to various vegetable crops; d) demonstrate if a direct relationship between the presence of contaminants in the environment, in agricultural products and, finally, in human matrices (hair, urine, blood) could be inferred.

Within the framework of the project, concentration of potentially toxic elements (PTE) and, in a lesser extent, persistent organic pollutants (POPs) were determined in $\sim 9000$ samples of top- and bottom-soils, $\sim 1200$ samples of groundwaters, $\sim 2500$ samples of agricultural products and $\sim 4200$ samples of biological matrices. Beside, seasonally, air and bulk deposition samples were collected from 150 passive air samplers and 50 deposimeters, respectively, over 7 seasons.

The obtained results showed that most of contamination due to PTEs and POPs is concentrated in Neapolitan Metropolitan Area (NMA) and in Sarno River basin. On the other hand, in contrast to what is publicly perceived, the territory between the provincial borders of Caserta and Naples, known as "Land of Fires", resulted only marginally interested by anomalous occurrence of PTMs and POPs in some spatially limited areas. Further, it was found that agricultural crops of "Land of Fires" were generally not enriched in PTEs

In general, we determined that in Campania urbanized areas play a key role as source of POPs rather than extra-urban areas (i.e. "Land of Fire"). 